\title{
Genome-wide analysis of transposable elements and tandem repeats in the compact placozoan genome
}

\author{
Shi Wang*1,2, Lingling Zhang ${ }^{2}$, Eli Meyer ${ }^{1}$ and Zhenmin Bao*3
}

\begin{abstract}
:The placozoan Trichoplax adhaerens has a compact genome with many primitive eumetazoan characteristics. In order to gain a better understanding of its genome architecture, we conducted a detailed analysis of repeat content in this genome. The transposable element (TE) content is lower than that of other metazoans, and the few TEs present in the genome appear to be inactive. A new phylogenetic clade of the gypsy-like LTR retrotransposons was identified, which includes the majority of gypsy-like elements in Trichoplax. A particular microsatellite motif (ACAGT) exhibits unexpectedly high abundance, and also has strong association with its nearby genes.
\end{abstract}

Reviewers: This article was reviewed by Dr. Jerzy Jurka and Dr. I. King Jordan.

\section{Findings}

Placozoans are arguably the simplest free-living multicelluar animals, and may represent an extant example of the ancestral metazoan body plan [1]. A recent comprehensive phylogenetic study suggests that placozoans are basal relatives to all other non-Bilaterian animals [[2], but see [3]]. It has been suggested that the placozoan Trichoplax adhaerens is an excellent model for the study of early evolution of metazoans $[4,5]$. The recent analysis of the Trichoplax genome has revealed a lack of the frequent intron loss and genomic rearrangement that characterize other small metazoan genomes (e.g. flies and worms), and many structural aspects (e.g. introns, local gene order and larger-scale linkages) are thought to represent ancestral eumetazoan characteristics [1]. In order to gain a better understanding of the evolution of the Trichoplax genome architecture, it may be interesting to investigate the abundance and types of repetitive sequences in the Trichoplax genome, because repetitive sequences, especially transposable elements (TEs), are major evolutionary contribu-

\footnotetext{
*Correspondence: swang@mail.utexas.edu ,zmbao@ouc.edu.cn

1 Section of Integrative Biology, School of Biological Sciences, University of Texas at Austin, 1 University Station C0930, Austin, TX 78712, USA

1 Section of Integrative Biology, School of Biological Sciences, University of Texas at Austin, 1 University Station C0930, Austin, TX 78712, USA Full list of author information is available at the end of the article
}

tors that drive genome evolution by enhancing genome plasticity [6-8].

We adopted both homology-based and ab initio methods to search for putative TEs. A full description of these methods is available in additional file 1 . The $\sim 95 \mathrm{Mb}$ Trichoplax genome sequence was compared with the Repbase database 14.04 [9] using tblastx [10] with an evalue threshold of $10^{-4}$, revealing 139 putative elements with significant similarity to known TEs (available in additional file 2). These elements accounted for only $0.13 \%$ of the Trichoplax genome, which is much lower than TE content of other small metazoan genomes (Table 1). The low TE content of the Trichoplax genome reported here is consistent with the previous report [1], in which 665 putative TEs were identified using the RepeatMasker program although no data curation and TE characterization were performed. The scarcity of TEs may explain why this genome has undergone the fewest rearrangement among metazoan genomes [1], because TEs are generally believed to be the major facilitators of this process [6-8]. The putative TEs we identified included representatives of three major TE classes: long terminal repeat (LTR) retrotransposons, non-LTR retrotransposons and DNA transposons (Table 2). DNA transposons were the most abundant TEs in the Trichoplax genome, and included diverse superfamilies such as helitron, piggyBac, hAT, mariner/Tc1, polinton and 
Table 1: Summary of repeat content in the placozoan and other metazoan genomes.

\begin{tabular}{|c|c|c|c|c|c|}
\hline & $\begin{array}{c}\text { Placozoan } \\
\text { (T. adhaerens) }\end{array}$ & $\begin{array}{l}\text { Nematode } \\
\text { (C. elegans) }\end{array}$ & $\begin{array}{c}\text { Arthropod } \\
\text { (D. melanogaster) }\end{array}$ & $\begin{array}{c}\text { Chordate } \\
\text { (T. nigroviridis) }\end{array}$ & $\begin{array}{l}\text { Chordate } \\
\text { (H. sapiens) }\end{array}$ \\
\hline Genome size (Mb) & 104 & 97 & 180 & 340 & 3,200 \\
\hline Gene no. & $\sim 11,500$ & $\sim 19,000$ & $\sim 13,600$ & $\sim 22,400$ & $\sim 31,000$ \\
\hline $\begin{array}{l}\text { Transposable } \\
\text { element (TE) }\end{array}$ & $0.13 \%$ & $6.5 \%$ & $3.1 \%$ & $0.9 \%$ & $44.4 \%$ \\
\hline $\begin{array}{l}\text { LTR } \\
\text { retrotransposon }\end{array}$ & $0.04 \%$ & $0.0 \%$ & $1.5 \%$ & $0.1 \%$ & $8.1 \%$ \\
\hline $\begin{array}{l}\text { Non-LTR } \\
\text { retrotransposon }\end{array}$ & $0.003 \%$ & $0.4 \%$ & $0.7 \%$ & $0.8 \%$ & $33.4 \%$ \\
\hline DNA transposon & $0.09 \%$ & $5.3 \%$ & $0.7 \%$ & $0.0 \%$ & $2.8 \%$ \\
\hline Active TE & None & Yes & Yes & Yes & Yes \\
\hline Tandem repeat & $2.7 \%$ & $2.7 \%$ & $3 \%$ & $4.5 \%$ & $3 \%$ \\
\hline Microsatellite & $0.2 \%$ & $0.2 \%$ & $0.5 \%$ & $3.2 \%$ & $1.5 \%$ \\
\hline Major SSR motif & ACAGT & AG & $A C$ & A & $A C$ \\
\hline $\begin{array}{l}\text { Minisatellite/ } \\
\text { Satellite }\end{array}$ & $2.4 \%$ & $2.5 \%$ & $2.5 \%$ & $1.3 \%$ & $1.5 \%$ \\
\hline Reference & [1] & {$[18,24,25]$} & {$[18,25-28]$} & [29-31] & [25] \\
\hline
\end{tabular}

$M u D R$. LTR retrotransposons were the second most abundant TEs in the Trichoplax genome, and the majority of these belonged to the gypsy superfamily. Non-LTR retrotransposons were rare in the Trichoplax genome, and were represented by only three superfamilies. Most of the elements we identified contained frequent stop codons or/and frameshifts in the coding regions, and appeared fragmented. Putative complete open reading frames (ORFs) were identified in only 6 elements (additional file 2), of which half belonged to $h A T$, and half to mariner $/ T c 1$. However, further investigation revealed that these lacked terminal inverted repeats (TIRs), and that each was present as only a single copy in the genome (blastn, e $\leq 10^{-4}$ ). Overall, none of the elements we identified contain all the features required for functional activ- ity in the Trichoplax genome, a finding that is also supported by the complete lack of putative TEs in the 14,572 expressed sequence tags (ESTs) available for $T$. adhaerens (tblastx against the Repbase database 14.04, e $\left.\leq 10^{-4}\right)$. In an effort to identify novel TEs in the Trichoplax genome, we conducted searches for LTR retrotransposons and miniature inverted-repeat transposable elements (MITEs) based on their respective structural features. Ten putative LTR retrotransposons were identified using the LTR_FINDER program [11], and none of them showed protein homology to known LTR retrotransposons or other TEs. Moreover, none of them were present in the genome with more than two copies, so it's unlikely that these are true LTR retrotransposons. MITEs belong to nonautonomous DNA transposons, and 
Table 2: Classification of transposable elements (TEs) in the Trichoplax genome.

\begin{tabular}{|c|c|c|}
\hline TE Superfamily & Counts & Matching Length (bp) \\
\hline \multicolumn{3}{|c|}{ LTR Retrotransposon } \\
\hline Gypsy & 53 & 38727 \\
\hline BEL & 1 & 903 \\
\hline DIRS & 1 & 633 \\
\hline ERV1 & 1 & 480 \\
\hline \multicolumn{3}{|c|}{ Non-LTR Retrotransposon } \\
\hline R4 & 1 & 1395 \\
\hline Jockey & 1 & 867 \\
\hline Penelope & 1 & 429 \\
\hline \multicolumn{3}{|c|}{ DNA Transposon } \\
\hline Helitron & 17 & 12820 \\
\hline PiggyBac & 16 & 14676 \\
\hline hAT & 14 & 16035 \\
\hline Mariner/Tc1 & 11 & 9098 \\
\hline Polinton & 10 & 14820 \\
\hline MuDR & 6 & 3498 \\
\hline Harbinger/PIF & 3 & 2479 \\
\hline ISL2EU & 2 & 6071 \\
\hline Chapaev & 1 & 2036 \\
\hline Total & 139 & 124967 \\
\hline
\end{tabular}


are usually present in eukaryotic genomes in very high copy numbers [12]. MITE analysis was carried out using the MUST program [13]. We considered a potential MITE family by requiring at least 3 elements in this family with the same perfect TIRs and target site duplications (TSDs). MITEs turned out to be quite rare in the genome, with only a single family represented by $\sim 20$ copies altogether. This is anticipated since no functional autonomous TEs, upon which MITEs rely for their transposition and persistence, were identified in the Trichoplax genome. Indeed, in a search of Trichoplax TEs in the Repbase database [9], only five putative nonautonomous DNA transposons with low copy numbers have been reported so far [14].

Further investigation of phylogenetic relationships within each TE superfamiliy was generally hampered by the low abundance and degenerated state of TEs representing the identified TE superfamilies. However, eleven gypsy-like elements (Ta1-11) shared a recognizable reverse transcriptase (RT) region, thus enabling investigation of their phylogenetic relationships. No outgroup was included in the phylogenetic analysis because we could not obtain a well-defined phylogeny based on the limited RT protein sequences when outgroup sequences were added. To date, nine phylogenetic clades have been identified in the gypsy group $[15,16]$. Our phylogenetic analysis of placozoan gypsy-like elements revealed a new clade (named Tag), which was formed by eight elements, Ta1-8 (Fig. 1, additional file 3). Since most of placozoan gypsy-like elements belong to the Tag clade, and this new clade has not been identified before in other metazoan genomes, this may suggest that much of the diversity among gypsy-like clades emerged after the divergence of Trichoplax from other metazoan lineages. It is also possible that Tag clade may represent an ancestral gypsy clade which was still preserved in the Trichoplax genome. Further identification and characterization of full-length elements belonging to the Tag clade from other eukaryotic organisms may provide new insights into the origin and diversification of the gypsy-like LTR retrotransposons. Two elements ( $\mathrm{Ta} 9$ and $\mathrm{Ta} 10$ ) were grouped into the previously identified Mag clade [15]. The element Ta11 may represent another new clade, but more data from other animal genomes are needed to verify this.

In contrast to the extremely low TE content, tandem repeats including microsatellites and minisatellites, represented a major source of repeat sequences, and accounted for $2.7 \%$ of the Trichoplax genome. The tandem repeat content of the Trichoplax genome is thus comparable to those of other small metazoan genomes, and even the huge human genome (Table 1). Using the SciRoko program [17], 11,697 microsatellites (repeat units within 1 6 bp) were identified in the Trichoplax genome based on criteria described in a previous study (perfect repeats $>12$ bp long), which had surveyed and analyzed the abundance and distribution of microsatellites in diverse eukaryotic taxonomic groups [18]. Surprisingly, we found that pentanucleotides were the most abundant repeat type in the Trichoplax genome, and accounted for $61.8 \%$ (i.e. 7233) of total microsatellites (additional file 4). This is unusual because mono-, di- and trinucleotides are usually the most abundant repeat types of microsatellites investigated in eukaryotic genomes so far [18-21]. To our knowledge, this finding represents the first report of pentanucleotide as the most abundant repeat type of microsatellites in a eukaryotic genome. More interestingly, further investigation revealed that majority $(78 \%)$ of the pentanucleotide repeats were accounted for by a single motif (ACAGT). If this particular motif is discounted, other pentanucleotides only account for $13.8 \%$ of total microsatellites, which is slightly lower than the abundance (14.2\%) of dinucleotides, suggesting the unusual pentanucleotide abundance in the Trichoplax genome is driven by the highly abundant ACAGT motif. Investigation of microsatellite abundance in the Trichoplax EST sequences revealed that as expected, trinucleotides, other than pentanucleotides, were the most abundant repeat type (52.4\%), although ACAGT motif was still the most abundant pentanucleotide motif. Many studies have shown that microsatellites can serve as transcription factor binding sites (TFBSs) to regulate gene expression [for a review, see [19]]. In order to evaluate if ACAGT motif could be a potential TFBS, we investigated the association pattern of ACAGT motif and its downstream nearby genes (Fig. 2, more info in additional file 5). Strikingly, $54 \%$ and $85 \%$ of ACAGT motif located within $1 \mathrm{~kb}$ and $5 \mathrm{~kb}$ upstream of nearby genes respectively, suggesting the potential role of ACAGT motif in regulation of nearby gene expression. Further gene ontology (GO) enrichment analysis of ACAGT associated genes (GO term level $=6$ and distance threshold $=5 \mathrm{~kb}$ ) revealed that 58 genes significantly enriched in the GO term of translation (adjusted $p$ $<0.021$ ), and 155 in the protein modification process (adjusted $p<0.0064$ ). Most of the translation genes are ribosomal proteins, and most of the protein modification genes are kinases. Kinases are known to regulate the majority of cellular pathways, especially those involved in signal transduction. Previous study has shown that Trichoplax genome encodes a rich array of transcription factors and signaling pathways that are typically associated with eumetazoan developmental patterning and celltype specification [1]. It would therefore be interesting to explore the potential roles of ACAGT motif in these biological processes in the future.

Minisatellites (repeat units usually within 7 2000 bp) were detected using the program Tandem Repeat Finder 4.03 [22]. In total, 9208 minisatellites with repeat units 


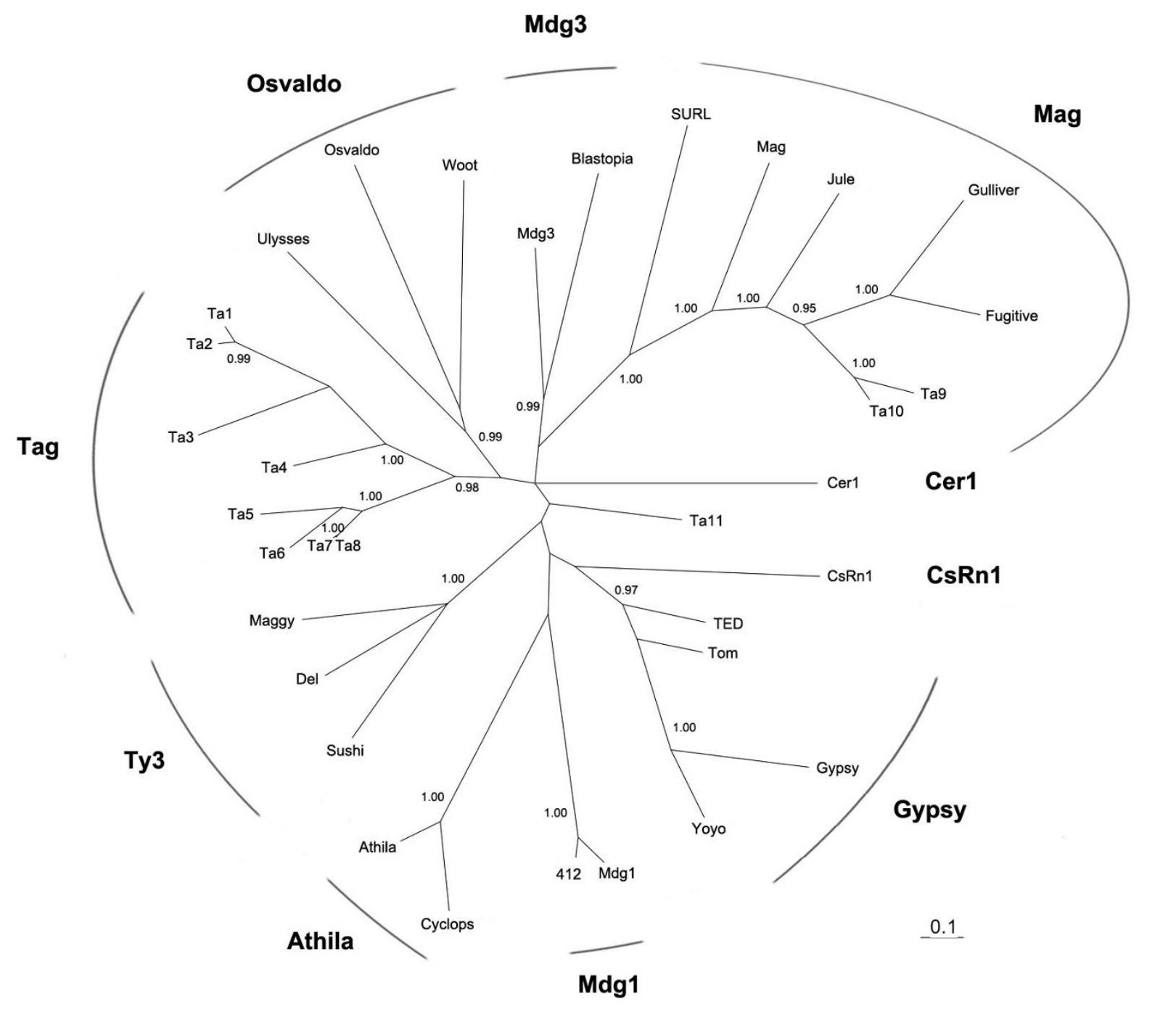

Figure 1 Phylogenetic relationships of T. adhaerens gypsy-like elements (Ta1-11) and other elements from the gypsy group based on a Bayesian analysis of reverse transcriptase (RT) protein sequences. Posterior probability exceeding 0.90 are shown. The sequences of most elements are derived from the EMBL online database [EMBL:DS36733] [15], while the others are as follows: CsRn1 [GenBank:AAK07487], Gulliver [GenBank:AF243513], Fugitive [GenBank:BK005226] and Jule [GenBank:AF278691]. RT sequences were aligned using the ClustalW method [32]. The protein alignment is available in the additional file 3. Phylogenetic analysis was performed with the program MrBayes 3.1 [33]. The appropriate model of evolution was identified as WAG+G+l [34] using the MCMC model-jumping method. The MCMC chain was run for 1,000,000 generations with a sample frequency of 200. In total, 5000 trees were produced, of which the first 4500 were discarded as burn-in while summarizing the data.

ranging from $7 \mathrm{bp}$ to $1204 \mathrm{bp}$ were identified in the Trichoplax genome, and accounted for $2.4 \%$ of the genome size. In general, the smaller the repeat unit, the higher the repeat abundance (additional file 6a). Minisatellites with repeat units ranging from $7 \mathrm{bp}$ to $25 \mathrm{bp}$ contained highly abundant of repeats (usually $>100$ repeats). Similar distribution patterns have previously been observed in a scallop genome [23]. The average copy number of these repeats was generally low in the Trichoplax genome $(<4$ copies for $94 \%$ of minisatellite repeat types) (additional file 6b), which may also account for the lack of frequent genomic rearrangements in the Trichoplax genome.

In summary, we conducted a detailed analysis of repeat content in the Trichoplax genome. The TEs in the Trichoplax genome are scarce and apparently lack functional activity. A new phylogenetic clade (Tag) of the gypsy-like LTR retrotransposons was identified. The unexpectedly high abundance of ACAGT motif in the Trichoplax genome represents an intriguing topic for future investi- 


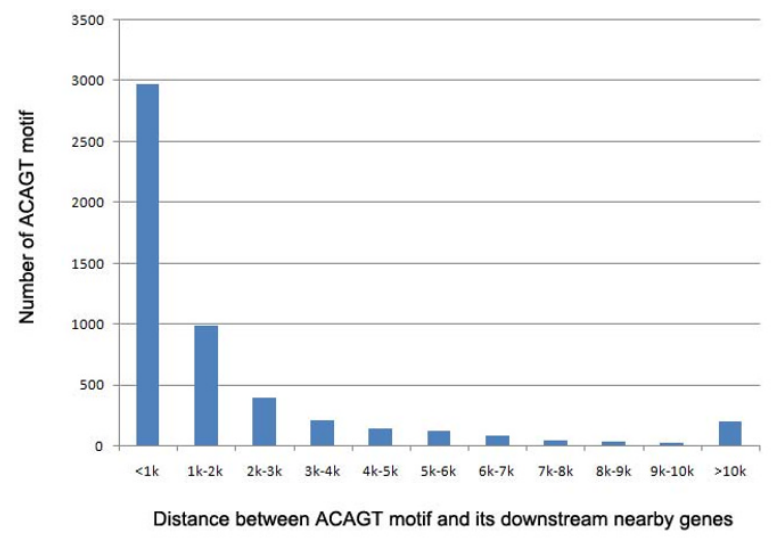

Figure 2 Association pattern between ACAGT motif and its downstream nearby genes.

gations of its potential roles in animal development and genome evolution.

\section{Reviewers' comments}

Reviewer 1 (Dr. Jerzy Jurka, Genetic Information Research Institute, USA)This paper analyzes repetitive DNA in an interesting metazoan. Of particular interest is the predominance of the (ACAGT)n microsatellite. However, it is difficult to evaluate the analysis of TEs. The authors should include all the identified TE sequences in a supplemental file.

Authors' response: Done. All identified TE sequences and annotations are included in the additional file 2.

Furthermore, they should include analysis of non-autonomous elements if they are present. The paper needs a second review.

Authors' response: We have used the MUST program to search for miniature inverted-repeat transposable elements (MITEs), which belong to nonautonomous DNA transposons, and are usually present in eukaryotic genomes in very high copy numbers. We considered a potential MITE family by requiring at least 3 elements in this family with the same perfect TIRs and TSDs. MITEs turned out to be quite rare in the genome, with only a single family represented by $\sim 20$ copies altogether. This is anticipated since no functional autonomous TEs, upon which MITEs rely for their transposition and persistence, were identified in the Trichoplax genome.

Reviewer 1's second review:

Here are putative non-autonomous DNA transposons published in October issue of Repbase Reports.
Authors' response: We appreciate the reviewer kindly providing this information. It is now mentioned and cited in the revised MS.

Reviewer 2 (Dr. I. King Jordan, Georgia Institute of Technology, USA)This manuscript describes an analysis of the short tandem repeat and long interspersed repeat, i.e. transposable element (TE), content of the Trichoplax adhaerens genome. An analysis of the repeat content of this genome is potentially interesting because the organism has a small compact genome and it occupies a basal position in the eukaryotic phylogenetic tree. Indeed, it has been claimed previously that Trichoplax likely resembles an ancestral eukaryotic genome. As such, genomic studies of this organism may reveal insight into the origin and evolution of eukaryotic genomes. The paper reports on a straightforward analysis, and it is well written and easy to follow. However, it is not clear what truly new or relevant insight into eukaryotic genome evolution is provided by these data. In addition, the methods used to search for repeats are not sufficiently rigorous to justify the conclusions that are made regarding the repeat content of the genome. I elaborate on these points and provide more specific comments below.

The most pressing point here is related to the authors' contention that Trichoplax represents an ancestral eukaryotic genome and therefore its repeat content can be understood to resemble that of the earliest eukaryotes. The problem is that repeats are notoriously dynamic genomic elements. Tandem repeats are highly unstable, and TEs are typically the most lineage-specific sequences in eukaryotic genomes. In fact, TEs are known quickly evolve beyond the ability to be recognized with homology based methods. Thus, the interspersed repeats that exist in the genome today, in particular those that can be found by the methods used here, were certainly not around in at the origin of the eukaryotes. Indeed, many of the TEs identified here seem to have been recently acquired and are rapidly decaying. It does not seem possible, based on the analysis of a single genome as reported here, to determine whether the low repeat content of Tichoplax is due to low repeats in the eukaryotic ancestor or secondary loss of repeats and genome streamlining over time.

Authors' response: We agree with this comment. The corresponding discussion has been removed from the MS.

Given the small size of the Trichoplax genome, along with its basal phylogenetic position in the eukaryotic tree, it is perhaps unsurprising that the authors turn up so few TEs. However, the overall lack of TEs reported here places a burden of proof on the authors that has not been met. It is up to them to demonstrate that they have exhaustively searched the genome sequence for TEs using a wide variety of available methods. The report indicates that BLAST 
was used to search for encoded protein sequences and a single ab initio method was used to search for MITEs. There are of course numerous tools available to search for TEs and repeats in genome sequences (e.g. see Bergman and Quesneville 2007 Brief Bioinform 8: 382). In fact, the most rigorous efforts at genome annotation now involve pipelines that combine the use of many tools - including both homology based detection methods based on comparisons between genome sequences and TE consensus sequences and $a b$ initio methods that rely on specific structural features of the elements (e.g. see Estill and Bennetzen 2009 Plant Methods 5: 8; Quesneville et al. 2005 PLoS Comput Biol. 1: 166). A deeper analysis of the repeat content of this genome would require such a combined approach.

Authors' response: The low TE content of the Trichoplax genome has been first reported in the previous study [1] where 665 putative TEs were identified using the RepeatMasker program although no data curation and TE characterization were performed. In a search for nonautonomous TEs in the Trichoplax genome, only five putative nonautonomous DNA transposons with low copy numbers have been identified so far [14]. Besides our tblastx comparison and MITE analysis, we also performed an additional analysis to search for novel LTR retrotransposons based on their structural features. Ten putative LTR retrotransposons were identified using the LTR_FINDER program [11], and none of them showed protein homology to known LTR retrotransposons or other TEs. Moreover, none of them were present in the genome with more than two copies, so it looks unlikely that these elements are true LTR retrotransposons. Overall, we conclude that the TE content of the Trichoplax genome is indeed very low, and that this observation is robust across a variety of methods.

Only a cursory description of the methods used to search for repeats are provided in the body of the manuscript. An additional description of the methods was provided by the authors upon request. These methodological details need to be included with the submission (perhaps as a supplement?) so that interested readers can more carefully evaluate the research design and the results.

Authors' response: We have now provided a complete description of methods in the additional file 1.

There are several statements regarding the relevance and the impact of the findings that are never substantiated or followed up on. For instance, in the abstract the authors state that "the unexpected abundance of [the ACAGT] motif makes this an attractive target for future studies into animal development and genome evolution." And in the body of the manuscript they claim that "Identification of the new phylogenetic clade, Tag may provide new insights into the origin and diversification of the gypsy-like LTR retrotransposons in metazoan genomes. "It is not clear what either of these strictly descriptive findings regarding Trichoplax genome repeats reveals about the organisms evolution or development. How does the abundance of a short tandem relate to the development of this organism? What does the discovery of a new gypsy clade, nested squarely within the diversity of existing gypsy-like sequences, tell us about the origin and diversification of the group?

Authors' response: 1) We added more analyses to explore the potential functions of ACAGT motif. We have rewritten the discussion in the light of new results. See the following:

Many studies have shown that microsatellites can serve as transcription factor binding sites (TFBSs) to regulate gene expression [for a review, see [19]]. In order to evaluate if ACAGT motif could be a potential TFBS, we investigated the association pattern of ACAGT motif and its downstream nearby genes (Fig. 2, more info in additional file 5). Strikingly, $54 \%$ and $85 \%$ of ACAGT motif located within $1 \mathrm{~kb}$ and $5 \mathrm{~kb}$ upstream of nearby genes respectively, suggesting the potential role of ACAGT motif in regulation of nearby gene expression. Further gene ontology (GO) enrichment analysis of ACAGT associated genes ( $\mathrm{GO}$ term level $=6$ and distance threshold $=5 \mathrm{~kb}$ ) revealed that 58 genes significantly enriched in the GO term of translation (adjusted $\mathrm{p}<0.021$ ), and 155 in the protein modification process (adjusted $\mathrm{p}<0.0064$ ). Most of the translation genes are ribosomal proteins, and most of the protein modification genes are kinases. Kinases are known to regulate the majority of cellular pathways, especially those involved in signal transduction. Previous study has shown that Trichoplax genome encodes a rich array of transcription factors and signaling pathways that are typically associated with eumetazoan developmental patterning and cell-type specification [1]. It would therefore be interesting to explore the potential roles of ACAGT motif in these biological processes in the future.

2) The phylogenetic tree of gypsy group presented in this study is an unrooted tree, and thus no ancestry information could be inferred from this tree. No outgroup was included in the phylogenetic analysis because we could not obtain a well-defined phylogeny based on the limited RT protein sequences when outgroup sequences were added. However, since most of placozoan gypsy-like elements belong to the Tag clade, and this new clade has not been identified before in other metazoan genomes, this may suggest that much of the diversity among gypsy-like clades emerged after the divergence of Trichoplax from other metazoan lineages. It is also possible that Tag clade may represent an ancestral gypsy clade which was still preserved in the Trichoplax genome. Further identification and characterization of full-length elements belonging to the Tag clade from other eukaryotic organisms would help clarify this situation, and may also provide 
new insights into the origin and diversification of the gypsy-like LTR retrotransposons.

In summary, given the abundance of new genomes that are constantly being sequenced, one has to wonder about the need to publish a description of the repeat content, or other specific aspects of genome architecture, in each case. It would seem that to justify such an exercise, the work must provide some fundamental new insight or at the very least clearly address a specific hypothesis. This report does not meet those standards, and so I am left to wonder as to the potential impact and overall relevance of the work.

Authors' response: In the revised MS, we present new analyses and discussion to expand on the previous statements. We feel that the unusual features of repeat content in Trichoplax are noteworthy, and that our analysis provides a useful review of those features and calls attention to a particular sequence motif that appears to be significantly associated with translation and signaling genes. We hope the reviewer will find that our MS has been improved enough to justify its publication as a Discovery Note.

Reviewer 2's second review:

I am satisfied with the authors' responses to my comments and support publication of the revised manuscript as a Discovery Note in Biology Direct.

\section{Additional material}

Additional file 1 Supplementary methods.

Additional file 2 Summary of 139 putative TEs detected in the Trichoplax genome.

Additional file 3 An alignment of Ta1-11 and other gypsy-like reverse transcriptase (RT) protein sequences using the ClustalW method (fasta and "aligned" formats).

Additional file $\mathbf{4}$ Characterization of microsatellites in the genome and EST sequences of the placozoan Trichoplax adhaerens.

Additional file 5 Summary of ACAGT motif and its downstream nearby genes.

Additional file 6 The distribution of abundance and average copy number in minisatellites.

\section{Abbreviations}

TE: transposable element; LTR: long terminal repeat; ORF: open reading frame; TIR: terminal inverted repeat; EST: expressed sequence tag; MITE: miniature inverted-repeat transposable element; TSD: target site duplication; RT: reverse transcriptase; TFBS: transcription factor binding site; GO: gene ontology.

\section{Competing interests}

The authors declare that they have no competing interests.

\section{Authors' contributions}

SW conceived the study. SW, LZ and EM conducted bioinformatic analyses. SW, LZ, EM and ZB discussed and interpreted the results. SW, EM and ZB drafted the manuscript.

\section{Acknowledgements}

We thank Dr. Fengfeng Zhou (University of Georgia) for the help in the MITE analysis. We also thank Dr. Mikhail V. Matz (University of Texas at Austin) for useful comments on the early draft of this manuscript.

\section{Author Details}

'Section of Integrative Biology, School of Biological Sciences, University of Texas at Austin, 1 University Station C0930, Austin, TX 78712, USA, 2Waggoner Center for Alcohol and Addiction Research, University of Texas at Austin, 1 University Station A4800, Austin, TX 78712, USA and ${ }^{3}$ Key Laboratory of Marine Genetics and Breeding, Ocean University of China,5 Yushan Road, Qingdao 266003, China

Received: 8 April 2010 Accepted: 15 April 2010

Published: 15 April 2010

\section{References}

1. Srivastava M, Begovic E, Chapman J, Putnam NH, Hellsten U, Kawashima T, Kuo A, Mitros T, Salamov A, Carpenter ML, Signorovitch AY, Moreno MA, Kamm K, Grimwood J, Schmutz J, Shapiro H, Grigoriev IV, Buss LW, Schierwater B, Dellaporta SL, Rokhsar DS: The Trichoplax genome and the nature of placozoans. Nature 2008, 454:955-960

2. Schierwater B, Eitel M, Jakob W, Osigus H-J, Hadrys H, Dellaporta SL, Kolokotronis S-O, DeSalle R: Concatenated analysis sheds light on early metazoan evolution and fuels a modern "Urmetazoon" hypothesis. PloS Biol 2009, 7:e1000020.

3. Dunn CW, Hejnol A, Matus DQ, Pang K, Browne WE, Smith SA, Seaver E, Rouse GW, Obst M, Edgecombe GD, Sorensen MV, Haddock SHD, Schmidt-Rhaesa A, Okusu A, Kristensen RM, Wheeler WC, Martindale MQ Giribet G: Broad phylogenomic sampling improves resolution of the animal tree of life. Nature 2008, 452:745-749.

4. Schierwater B: My favorite animal, Trichoplax adhaerens. BioEssays 2005, 27:1294-1302.

5. Signorovitch AY, Dellaporta SL, Buss LW: Molecular signatures for sex in the Placozoa. Proc Natl Acad Sci USA 2005, 102:15518-15522.

6. Kazazian HH Jr: Mobile elements: drivers of genome evolution. Science 2004, 303:1626-1632.

7. Wessler SR: Eukaryotic transposable elements: teaching old genomes new tricks. In The Implicit Genome Edited by: Caporale L. New York: Oxford University Press; 2006:138-165.

8. Jurka J, Kapitonov VV, Kohany O, Jurka MV: Repetitive sequences in complex genomes: structure and evolution. Annu RevGenomics Hum Genet 2007, 8:241-259.

9. Jurka J, Kapitonov WV, Pavlicek A, Klonowski P, Kohany O, Walichiewicz J: Repbase Update, a database of eukaryotic repetitive elements. Cytogenet Genome Res 2005, 110:462-467.

10. Altschul SF, Gish W, Miller W, Myers EW, Lipman DJ: Basic local alignment search tool. J Mol Biol 1990, 215:403-410.

11. Xu Z, Wang H: LTR FINDER: an efficient tool for the prediction of fulllength LTR retrotransposons. Nucleic Acids Res 2007, 35:W265-W268.

12. Feschotte $C$, Zhang $X$, Wessler SR: Miniature inverted-repeat transposable elements and their relationship with established DNA transposons. In Mobile DNA // Edited by: Craig N, Craigie R, Gellert M, Lambowitz A. WA: American Society of Microbiology Press; 2002:1147-1158.

13. Chen Y, Zhou F, Li G, Xu Y: MUST: A system for identification of miniature inverted-repeat transposable elements and applications to Anabaena variabilis and Haloquadratum walsbyi. Gene 2009, 436:1-7.

14. Jurka J: DNA transposons from Trichoplax adhaerens. Repbase Rep 2009, 9:2144-2148

15. Malik H, Eickbush TH: Modular evolution of the integrase domain in the Ty3/Gypsy class of LTR retrotransposons. J Virol 1999, 73:5186-5190.

16. Bae Y-A, Moon S-Y, Kong Y, Cho S-Y, Rhyu M-G: CsRn1, a novel active retrotransposon in a parasitic trematode, Clonorchis sinensis, discloses a new phylogenetic clade of Ty3/gypsy-like LTR retrotransposons. Mol Biol Evol 2001, 18:1474-1483.

17. Kofler R, Schlotterer C, Lelley T: SciRoKo: a new tool for whole genome microsatellite search and investigation. Bioinformatics 2007 23:1683-1685

18. Toth G, Gaspari Z, Jurka J: Microsatellites in different eukaryotic genomes: survey and analysis. Genome Res 2000, 10:967-981.

19. Li Y-C, Korol AB, Fahima T, Beiles A, Nevo E: Microsatellites: genomic distribution, putative functions and mutational mechanisms: a review. Mol Ecol 2002, 11:2453-2465. 
20. Ellegren H: Microsatellites: simple sequences with complex evolution. Nat Rev Genet 2004, 5:435-445.

21. Sharma PC, Grover A, Kahl G: Mining microsatellites in eukaryotic genomes. Trends Biotechnol 2007, 25:490-498.

22. Benson G: Tandem repeats finder: a program to analyze DNA sequences. Nucleic Acids Res 1999, 27:573-580.

23. Zhang L, Chen C, Cheng J, Wang S, Hu X, Hu J, Bao Z: Initial analysis of tandemly repetitive sequences in the genome of Zhikong scallop (Chlamys farreri Jones et Preston). DNA Seq 2008, 19:195-205.

24. The C. elegans Sequencing Consortium: Genome sequence of the nematode C. elegans: a platform for investigating biology. Science 1998, 282:2012-2018.

25. International Human Genome Sequencing Consortium: Initial sequencing and analysis of the human genome. Nature 2001 409:860-921.

26. Adams MD, et al:: The genome sequence of Drosophila melanogaster. Science 2000, 287:2185-2195.

27. Katti MV, Ranjekar PK, Gupta V: Differential distribution of simple sequence repeats in eukaryotic genome sequences. Mol Biol Evol 2001, 18:1161-1167.

28. Smith CD, Shu S, Mungall CJ, Karpen GH: The release 5.1 annotation of Drosophila melanogaster heterochromatin. Science 2007, 316:1586-1591.

29. Crollius HR, Jaillon O, Dasilva C, Ozouf-Costaz C, Fizames C, Fischer C, Bouneau L, Billault A, Quetier F, Saurin W, Bernot A, Weissenbach J: Characterization and repeat analysis of the compact genome of the freshwater pufferfish Tetraodon nigroviridis. Genome Res 2000, 10:939-949

30. Bouneau L, Fischer C, Ozouf-Costaz C, Froschauer A, Jaillon O, Coutanceau J-P, Korting C, Weissenbach J, Bernot A, Volff J-N: An active non-LTR retrotransposon with tandem structure in the compact genome of the pufferfish Tetraodon nigroviridis. Genome Res 2003, 13:1686-1695.

31. Jaillon O, et al:: Genome duplication in the teleost fish Tetraodon nigroviridis reveals the early vertebrate proto-karyotype. Nature 2004, 431:946-957.

32. Thompson JD, Higgins DG, Gibson TJ: CLUSTAL W: improving the sensitivity of progressive multiple sequence alignment through sequence weighting, position-specific gap penalties and weigh matrix choice. Nucleic Acids Res 1994, 22:4673-4680.

33. Huelsenbeck JP, Ronquist F: Bayesian inference of phylogenetic trees. Bioinformatics 2001, 17:754-755.

34. Whelan S, Goldman N: A general empirical model of protein evolution derived from multiple protein families using a maximum-likelihood approach. Mol Biol Evol 2001, 18:691-699.

\section{doi: 10.1186/1745-6150-5-18}

Cite this article as: Wang et al., Genome-wide analysis of transposable elements and tandem repeats in the compact placozoan genome Biology Direct 2010, 5:18

\section{Submit your next manuscript to BioMed Central} and take full advantage of:

- Convenient online submission

- Thorough peer review

- No space constraints or color figure charges

- Immediate publication on acceptance

- Inclusion in PubMed, CAS, Scopus and Google Scholar

- Research which is freely available for redistribution

Submit your manuscript at www.biomedcentral.com/submit
C Biomed Central 